\title{
MHD Pulsatile Flow through a Porous Medium
}

\author{
R. $\operatorname{Kumar}^{1 \dagger}$, B. G. Prasad ${ }^{2}$ \\ ${ }^{1}$ Department of Applied Mathematics, Birla Institute of Technology, Patna Campus, Patna-14, Bihar, India \\ ${ }^{2}$ Department of Mathematics, B.N. College, Patna University, Patna-4, Bihar, India \\ $\dagger$ Corresponding Author Email: rajnish.bitpatna@gmail.com
}

(Received May 7, 2012; accepted January 18, 2013)

\begin{abstract}
This paper develops a mathematical model with an aim to compute the analytic solution for the MHD pulsatile flow driven by an unsteady pressure gradient between permeable beds of a viscous incompressible Newtonian fluid saturated porous medium. The dimensionless governing equations for this investigation are solved analytically after separating into a steady part and an unsteady part. The influence of pertinent parameters on the flow is delineated and appropriate conclusions are drawn. The results presented in this work constitute an innovative way to describe correctly the boundary conditions at both the walls. Favorable comparisons with previously published work authenticate a remarkable accuracy of the results investigated in the paper. Mathematics Subject Classification 2010: 76D05, 76D10, 76S05, 76W05.
\end{abstract}

Keywords: Newtonian fluid, Pulsatile flow, Permeable beds, Porous medium, MHD.

\section{NOMENCLATURE}

$$
\begin{array}{ll}
x_{s} y & \text { Cartesian co-ordinates } \\
u & \text { velocity component in x-direction } \\
V & \text { suction/injection velocity } \\
k_{1} & \text { permeability of the lower bed } \\
k_{2} & \text { permeability of the upper bed } \\
\sigma_{1} & \left(=\frac{\hbar}{\sqrt{k_{1}}}\right) \text { dimensionless parameter } \\
\sigma_{2} & \left(=\frac{\hbar}{\sqrt{k_{2}}}\right) \text { dimensionless parameter } \\
u_{B 1} & \text { slip velocity at the lower bed } \\
u_{B 2} & \text { slip velocity at the upper bed } \\
Q_{1} & -\frac{k_{1}}{p}\left(\frac{\partial p}{\partial x}\right) \text { Darcy's velocity } \\
Q_{2} & -\frac{k_{2}}{p}\left(\frac{\partial p}{\partial x}\right) \text { Darcy's velocity } \\
D a & \left(=\frac{k}{\hbar^{2}}\right) \text {. Darcy number } \\
R & \text { Reynolds number }
\end{array}
$$

\section{INTRODUCTION}

The MHD (magnetohydrodynamics) flow through a porous medium has been a fervent topic of study owing to its wide ranging applications in a numbers of field such as the MHD generators and accelerators in geophysics, soil sciences, astrophysics, nuclear power

$\begin{array}{ll}N & \text { frequency parameter } \\ M & \text { Hartmann number } \\ Q & \text { mass flow } \\ p & \text { pressure } \\ \alpha & \text { slip parameter } \\ h & \text { width of the channel } \\ \rho & \text { density } \\ \omega & \text { frequency } \\ \mu & \text { coefficient of viscosity } \\ \tilde{\tau} & \text { shear stress } \\ \nu & \text { kinematic viscosity } \\ \sigma^{*} & \text { electrical conductivity } \\ B_{0} & \text { magnetic field }\end{array}$

reactors, biomedical engineering and physiological fluid dynamics for more than five decades.

A number of investigations concerning the MHD flows of fluid saturated porous medium are available in the literature. For example; Raptis et al. (1982,1983), Hassanien (1989), Aldoss (1996), Chamkha (1997), (1999) and Chamkha et al. (2000). 
An early classical study was conducted by Uchida (1956). It was shown in these studies that for large blood vessels, the flow of blood is principally Newtonian and therefore approximations of the NavierStokes equations are justified. When applied transverse to the direction of flow, magnetic fields have been shown to impede blood flow, since blood as a plasma suspension is electrically-conducting. In a pioneering work of Wang (1971), the pulsatile flow in a porous channel was studied. Earlier, Berman (1958) investigated the steady two-dimensional laminar viscous incompressible fluid flow driven by uniform injection (or suction) in a rectangular channel through permeable and stationary walls. Meanwhile, Beavers and Joseph (1967) in their experimental work on boundary conditions at a naturally permeable wall confirmed the existence of slip at the interface separating the flow in the channel and the permeable boundaries. Radhakrishnamacharya and Maiti (1977) have made an investigation of heat transfer to pulsatile fluid flow in a porous channel. Khodadadi (1991) treated analytically an oscillatory, rather than a pulsating, flow through a porous medium-filled channel bounded by two impermeable parallel plates. Kim et al. (1994) examined the qualitative behavior of the pulsating flow and heat transfer in a channel fully filled with porous media. Chandra and Prasad (1994) provide a small sample of the research in pulsatile flow problem with varying cross-section of tubes. In a very significant study of pulsatile flow between permeable beds by Vajravelu et al. (2003), it was shown that the maximum velocity is attained between the permeable beds and gradually the velocity decreases towards the upper permeable bed. Hassan and Das (2008) discussed laminar sinusoidal pulsating flow through a modeled arterial stenosis with a trapezoidal profile up to peak Reynolds number of 1000. Malathy and Srinivas (2008) simulated the pulsatile hydromagnetic flow between permeable beds. The study indicated that the maximum velocity is attained even at the lower permeable bed in the case of some specific choice of parameters.

The motivation of the present study stems from the above considerations, i.e., to augment velocity profile by the presence of porous media and the use of pulsation. The following strategy is pursued in the rest of the paper. In Section 2 and 3 the details of the mathematical formulation the problem are outlined in Cartesian coordinates. Section 4 contains the analytical result, both for the steady and unsteady state basic flow. Many deductions including mass flux and shear stress are analyzed in Section 5. Our results are presented pictorially and discussed in Section 6 and finally in Section 7 interesting conclusions have been pointed out.

\section{MATHEMATiCal Statement OF THE PROBLEM}

Consider Consider the fully developed laminar pulsatile flow between two permeable beds at $y=0$ and $y=h$ of a viscous conducting fluid through a porous medium. The fluid is assumed to be Newtonian and incompressible. A magnetic field of uniform strength $B_{0}$ is applied in the direction of normal to the beds and the induced magnetic field is neglected. The $\mathrm{x}$-axis is taken along the interface and the $y$-axis is taken normal to it. The porous medium regime and the permeable beds are homogeneous and isotropic. The fluid is injected into the channel from the lower permeable bed with a velocity $V$ and is sucked out into the upper permeable bed with the same velocity. The domain of interest is shown in Fig. 1. The flow is driven by pressure gradient which comprises a steady and a periodic fluctuating component in the axial direction, $\frac{1}{\rho} \frac{\partial p}{\partial x}=A+B e^{i \omega t}$, where $A$ and $B$ are constants and $\omega$ is the frequency of the pressure pulsations.

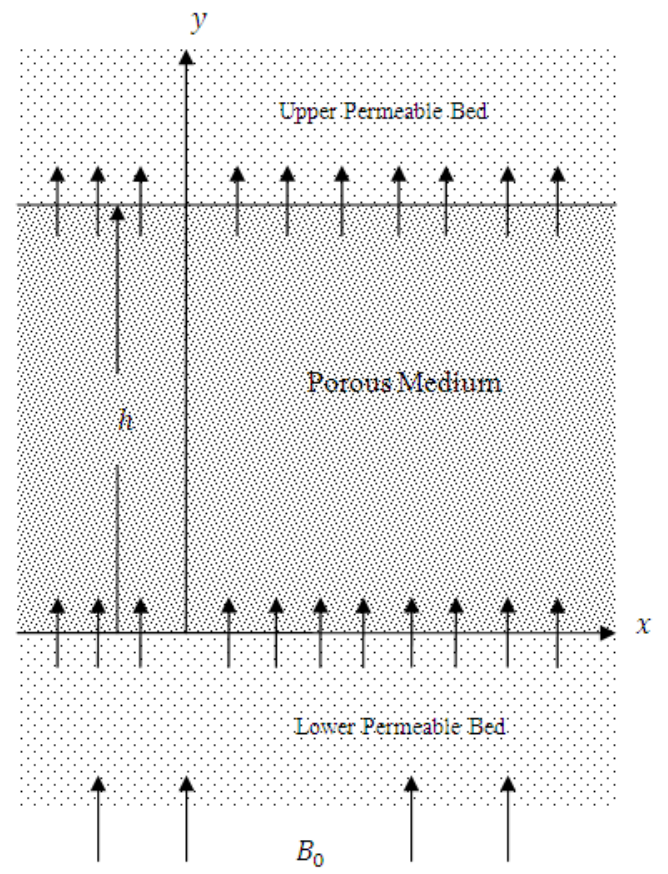

Fig. 1. The Physical Model and Coordinate system

The governing momentum equations from the incompressible Navier-Stokes equations can be shown to reduce to the following form for the MHD flow regime:

$\frac{\partial u}{\partial x}+\frac{\partial v}{\partial y}=0$

$\frac{\partial u}{\partial t}+V \frac{\partial u}{\partial y}=-\frac{1}{\rho} \frac{\partial p}{\partial x}+v \frac{\partial^{2} u}{\partial y^{2}}-\frac{\sigma^{*} B_{0}^{2} u}{\rho}-\frac{v}{k} u$

$0=\frac{\partial p}{\partial y}$

$u=u_{B 1}$ aty $=0$

$\frac{d u}{d y}=\frac{\alpha}{\sqrt{k_{1}}}\left(u_{B 1}-Q_{1}\right) a t y=0$

$u=u_{B 2}$ at $y=h$

$\frac{d u}{d y}=-\frac{\alpha}{\sqrt{k_{2}}}\left(u_{B 2}-Q_{2}\right) a t y=h$ 
R. Kumar and B. G. Prasad / JAFM, Vol. 7, No. 1, pp. 63-74, 2014.

The above boundary conditions are the commonly used slip conditions given by Beavers and Joseph (1967). They proposed that the tangential component of the normal stress of the flow in the conduit at the interface is proportional to the jump of the tangential velocity across the interface.

We separate all the above equations into a steady part and an unsteady part represented by a bar $(-)$ and a tilde $(\sim)$ respectively, they become

\subsection{Steady Part}

$\frac{\partial \bar{u}}{\partial x}=0$

$V \frac{d \bar{u}}{d y}=-A+v \frac{d^{2} \bar{u}}{d y^{2}}-\frac{\sigma^{*} B_{0}^{2} \bar{u}}{\rho}-\frac{v}{k} \bar{u}$

$\bar{u}=\bar{u}_{B 1}$ at $y=0$

$\frac{d \bar{u}}{d y}=\frac{\alpha}{\sqrt{k_{1}}}\left(\bar{u}_{B 1}-\bar{Q}_{1}\right) a t y=0$

$\bar{u}=\bar{u}_{B 2}$ at $y=h$

$\frac{d \bar{u}}{d y}=-\frac{\alpha}{\sqrt{k_{2}}}\left(\bar{u}_{B 2}-\bar{Q}_{2}\right) a t y=h$

where $\bar{Q}_{1}=-\frac{k_{1} \rho A}{\mu}, \bar{Q}_{2}=-\frac{k_{2} \rho A}{\mu}$

\subsection{Unsteady Part}

$\frac{\partial \tilde{u}}{\partial x}=0$

$\frac{\partial \tilde{u}}{\partial t}+V \frac{\partial \tilde{u}}{\partial y}=-B e^{i \omega t}+v \frac{\partial^{2} \tilde{u}}{\partial y^{2}}-\frac{\sigma^{*} B_{0}^{2} \tilde{u}}{\rho}-\frac{v}{k} \tilde{u}$

$\tilde{u}=\tilde{u}_{B 1} a t \quad y=0$

$\frac{d \tilde{u}}{d y}=\frac{\alpha}{\sqrt{k_{1}}}\left(\tilde{u}_{B 1}-\tilde{Q}_{1}\right) a t y=0$

$\tilde{u}=\tilde{u}_{B 2} a t y=h$

$\frac{d \tilde{u}}{d y}=-\frac{\alpha}{\sqrt{k_{2}}}\left(\tilde{u}_{B 2}-\tilde{Q}_{2}\right) a t y=h$

where $\tilde{Q}_{1}=-\frac{k_{1} \rho B}{\mu} e^{i \omega t}, \tilde{Q}_{2}=-\frac{k_{2} \rho B}{\mu} e^{i \omega t}$.

\subsection{Steady Part}

$$
\begin{aligned}
& \frac{\partial \tilde{u}}{\partial x}=0, \\
& \frac{\partial \tilde{u}}{\partial t}+V \frac{\partial \tilde{u}}{\partial y}=-B e^{i \omega t}+v \frac{\partial^{2} \tilde{u}}{\partial y^{2}}-\frac{\sigma^{*} B_{0}^{2} \tilde{u}}{\rho}-\frac{v}{k} \tilde{u}, \\
& \tilde{u}=\tilde{u}_{B 1} \quad \text { at } \quad y=0 \\
& \frac{d \tilde{u}}{d y}=\frac{\alpha}{\sqrt{k_{1}}}\left(\tilde{u}_{B 1}-\tilde{Q}_{1}\right) \quad \text { at } y=0 \\
& \tilde{u}=\tilde{u}_{B 2} \quad \text { at } \quad y=h \\
& \frac{d \tilde{u}}{d y}=-\frac{\alpha}{\sqrt{k_{2}}}\left(\tilde{u}_{B 2}-\tilde{Q}_{2}\right) \quad \text { at } \quad y=h
\end{aligned}
$$

where $\tilde{Q}_{1}=-\frac{k_{1} \rho B}{\mu} e^{i \omega t}, \tilde{Q}_{2}=-\frac{k_{2} \rho B}{\mu} e^{i \omega t}$.

\section{NON- DIMENSIONALIZATION}

In order to transform the above system of equations into a non-dimensionless form, use will be made of the following transformations:

\subsection{Steady Part}

$\bar{u}^{*}=\frac{\bar{u}}{\left(A_{1} h / V\right)}$,

$\bar{u}_{B 1}^{*}=\frac{\bar{u}_{B 1}}{\left(A_{1} h / V\right)}$,

$\bar{u}_{B 2}^{*}=\frac{\bar{u}_{B 2}}{\left(A_{1} h / V\right)}$,

$\bar{Q}_{1}^{*}=\frac{\overline{Q_{1}}}{\left(A_{1} h / V\right)}$,

$\bar{Q}_{2}^{*}=\frac{\overline{Q_{2}}}{\left(A_{1} h / V\right)}$,

$D a=\frac{k}{h^{2}}$,

$M^{2}=\frac{\sigma^{*} B_{0}^{2} h}{\rho V}$,

$x^{*}=\frac{x}{h}$,

$y^{*}=\frac{y}{h}$,

where $A_{1}=-A$.

\subsection{Unsteady Part}

$$
\begin{aligned}
& \tilde{u}^{*}=\frac{\tilde{u}}{\left(h^{2} B_{1} / v\right)}, \\
& \tilde{u}_{B 1}^{*}=\frac{\tilde{u}_{B 1}}{\left(h^{2} B_{1} / v\right)}, \\
& \tilde{u}_{B 2}^{*}=\frac{\tilde{u}_{B 2}}{\left(h^{2} B_{1} / v\right)}, \\
& t^{*}=\frac{t}{\left(h^{2} / v\right)}, \omega^{*}=\frac{\omega}{v / h^{2}}, \\
& D a=\frac{k}{h^{2}}, M{ }^{2}=\frac{\sigma^{*} B_{0}^{2} h}{\rho V}, \\
& \tilde{Q}_{1}^{*}=\frac{\tilde{Q}_{1}}{\left(h^{2} B_{1} / v\right)}, \\
& \tilde{Q}_{2}^{*}=\frac{\tilde{Q}_{2}}{\left(h^{2} B_{1} / v\right)}, \\
& x^{*}=\frac{x}{h}, y^{*}=\frac{y}{h},
\end{aligned}
$$


R. Kumar and B. G. Prasad / JAFM, Vol. 7, No. 1, pp. 63-74, 2014.

where $B_{1}=-B$.

Using above dimensionless quantities, Eq. (5)- Eq. (16) yield: Ignoring the asterisks( $(*)$ for clarity, we obtain the dimensionless governing equations as,

Steady Part:

$\frac{\partial \bar{u}}{\partial x}=0$

$\frac{d^{2} \bar{u}}{d y^{2}}-R \frac{d \bar{u}}{d y}-\left(M^{2} R+\frac{1}{D a}\right) \bar{u}=-R$

$\bar{u}=\bar{u}_{B 1}$ at $y=0$

$\frac{d \bar{u}}{d y}=\alpha \sigma_{1}\left(\bar{u}_{B 1}-\frac{R}{\sigma_{1}^{2}}\right) \quad$ at $\quad y=0$

$\bar{u}=\bar{u}_{B 2} \quad$ at $y=1$

$\frac{d \bar{u}}{d y}=-\alpha \sigma_{2}\left(\bar{u}_{B 2}-\frac{R}{\sigma_{2}^{2}}\right) \quad$ at $\quad y=1$

where $R=\frac{V h}{v}, \sigma_{1}=\frac{h}{\sqrt{k_{1}}}$, and $\sigma_{2}=\frac{h}{\sqrt{k_{2}}}$

Unsteady Part

$\frac{\partial \tilde{u}}{\partial x}=0$

$\frac{\partial^{2} \tilde{u}}{\partial y^{2}}-R \frac{\partial \tilde{u}}{\partial y}-\frac{\partial \tilde{u}}{\partial t}-\left(M^{2} R+\frac{1}{D a}\right) \tilde{u}=-e^{i \omega t}$

Assuming $\tilde{u}=\tilde{f}(y) e^{i \omega t}$, the above equation yields

$\tilde{f}^{\prime \prime}(y)-R \tilde{f}^{\prime}(y)-(i \omega) \tilde{f}-\left(M^{2} R+\frac{1}{D a}\right) \tilde{f}=-1$

$\Rightarrow \tilde{f}^{\prime \prime}(y)-R \tilde{f}^{\prime}(y)-\left(i \omega+M^{2} R+\frac{1}{D a}\right) \tilde{f}=-1$

and letting $\quad \tilde{u}_{B 1}=\tilde{f_{1}}(y) e^{i \omega t}, \tilde{u}_{B 2}=\tilde{f_{2}}(y) e^{i \omega t}$.

The boundary conditions reduce to

$$
\begin{aligned}
& \tilde{f}=\tilde{f_{1}} \quad \text { at } \quad y=0 \\
& \frac{d \tilde{f}}{d y}=\alpha \sigma_{1}\left(\tilde{f_{1}}-\frac{1}{\sigma_{1}^{2}}\right) \quad \text { at } \quad y=0 \\
& \tilde{f}=\tilde{f_{2}} \quad \text { at } \quad y=1 \\
& \frac{d \tilde{f}}{d y}=-\alpha \sigma_{2}\left(\tilde{f_{2}}-\frac{1}{\sigma_{2}^{2}}\right) \quad \text { at } \quad y=1
\end{aligned}
$$

The mathematical statement of the problem is now complete and embodies the solution of Eqs. (24) and (30b) subject to the above mentioned boundary conditions

\section{Analytical Solution}

\subsection{Steady part}

Using boundary conditions Eq. (25) - Eq. (28), we get the velocity field by solving Eq. (24) as

$$
\bar{u}=C_{1} e^{\alpha_{1} y}+C_{2} e^{\alpha_{2} y}+\frac{R}{\left(M^{2} R+\frac{1}{D a}\right)}
$$

where $\alpha_{1,2}=\frac{1}{2}\left[R \pm \sqrt{ }\left\{R^{2}+4\left(M^{2} R+\frac{1}{D a}\right)\right\}\right]$.

$C_{1}=\frac{1}{e^{\alpha_{2}}-e^{\alpha_{1}}}\left[-\bar{u}_{B 2}+e^{\alpha_{2}} \bar{u}_{B 1}-\frac{R}{\left(M^{2} R+\frac{1}{D a}\right)}\left(e^{\alpha_{2}}-1\right)\right]$

$C_{2}=\frac{1}{e^{\alpha_{2}}-e^{\alpha_{1}}}\left[\bar{u}_{B 2}-e^{\alpha_{1}} \bar{u}_{B 1}-\frac{R}{\left(M^{2} R+\frac{1}{D a}\right)}\left(1-e^{\alpha_{1}}\right)\right.$

and the slip velocities are given by

$\bar{u}_{B 1}=\frac{D_{2} E_{2}-D_{4} E_{1}}{D_{1} D_{4}-D_{3} D_{2}}$

$\bar{u}_{B 2}=\frac{D_{3} E_{1}-D_{1} E_{2}}{D_{1} D_{4}-D_{3} D_{2}}$

where

$$
\begin{aligned}
& D_{1}=\frac{\alpha_{1} e^{\alpha_{2}}-\alpha_{2} e^{\alpha_{1}}}{e^{\alpha_{2}}-e^{\alpha_{1}}}-\alpha \sigma_{1}, D_{2}=\frac{\alpha_{2}-\alpha_{1}}{e^{\alpha_{2}}-e^{\alpha_{1}}} \\
& D_{3}=\frac{\left(\alpha_{1}-\alpha_{2}\right) e^{\alpha_{1}+\alpha_{2}}}{e^{\alpha_{2}}-e^{\alpha_{1}}}, D_{4}=\frac{\alpha_{2} e^{\alpha_{2}}-\alpha_{1} e^{\alpha_{1}}}{e^{\alpha_{2}}-e^{\alpha_{1}}}+\alpha \sigma_{2} \\
& E_{1}=R\left[\frac{1}{\left(M^{2} R+\frac{1}{D a}\right)}\left\{\frac{\alpha_{2}\left(e^{\alpha_{1}}-1\right)}{e^{\alpha_{2}}-e^{\alpha_{1}}}-\frac{\alpha_{1}\left(e^{\alpha_{2}}-1\right)}{e^{\alpha_{2}}-e^{\alpha_{1}}}\right\}+\frac{\alpha}{\sigma_{1}}\right] \\
& E_{2}=R\left[\frac{1}{\left(M^{2} R+\frac{1}{D a}\right)}\left\{\frac{\alpha_{2} e^{\alpha_{2}}\left(e^{\alpha_{1}}-1\right)}{e^{\alpha_{2}}-e^{\alpha_{1}}}-\frac{\alpha_{1} e^{\alpha_{1}}\left(e^{\alpha_{2}}-1\right)}{e^{\alpha_{2}}-e^{\alpha_{1}}}\right\}-\frac{\alpha}{\sigma_{2}}\right]
\end{aligned}
$$

\subsection{Unsteady Part}

Using boundary conditions Eq. (31) to Eq. (34), we get the velocity field by solving Eq. (38b) as

$$
\begin{aligned}
& \tilde{f}(y)=A_{1} e^{\left(\beta_{1} y\right)}+A_{2} e^{\left(\beta_{2} y\right)}+\frac{1}{i N^{2}+M^{2} R+\frac{1}{D a}} \\
& \text { where } \beta_{1,2}=\frac{1}{2}\left[R \pm \sqrt{ }\left\{R^{2}+4\left(i N^{2}+M^{2} R+\frac{1}{D a}\right)\right\}\right] \\
& N^{2}=\omega
\end{aligned}
$$


R. Kumar and B. G. Prasad / JAFM, Vol. 7, No. 1, pp. 63-74, 2014.

$$
\begin{aligned}
& A_{1}=\frac{1}{e^{\beta_{2}}-e^{\beta_{1}}}\left[-\frac{e^{\beta_{2}}-1}{i N^{2}+M^{2} R+\frac{1}{D a}}-\tilde{f_{2}}+\tilde{f_{1}} e^{\beta_{2}}\right] \\
& A_{2}=\frac{1}{e^{\beta_{2}}-e^{\beta_{1}}}\left[\frac{e^{\beta_{1}}-1}{i N^{2}+M^{2} R+\frac{1}{D a}}+\tilde{f_{2}}-\tilde{f_{1}} e^{\beta_{1}}\right]
\end{aligned}
$$

The unsteady part of the velocity is expressed as

$\tilde{u}=\tilde{f}(y) e^{i \omega t}$

And the slip velocities are given by

$\tilde{u}_{B 1}=\tilde{f_{1}} e^{i \omega t}=\frac{P_{2} Z_{2}-Z_{1}\left(P_{4}+Q_{2}\right)}{\left(P_{1}+Q_{1}\right)\left(P_{4}+Q_{2}\right)-P_{3} P_{2}} e^{i \omega t}$,
$\tilde{u}_{B 2}=\tilde{f}_{2} e^{i \omega t}=\frac{P_{3} Z_{1}-Z_{2}\left(P_{1}+Q_{1}\right)}{\left(P_{1}+Q_{1}\right)\left(P_{4}+Q_{2}\right)-P_{3} P_{2}} e^{i \omega t}$

where

$$
\begin{aligned}
& P_{1}=\left(\beta_{1} e^{\beta_{2}}-\beta_{2} e^{\beta_{1}}\right), P_{2}=\beta_{2}-\beta_{1}, \\
& P_{3}=e^{\beta_{1}+\beta_{2}}\left(\beta_{1}-\beta_{2}\right), P_{4}=\left(\beta_{2} e^{\beta_{2}}-\beta_{1} e^{\beta_{1}}\right), \\
& R_{1}=\left(e^{\beta_{2}}-e^{\beta_{1}}\right), Q_{1}=\alpha \sigma_{1}\left(e^{\beta_{1}}-e^{\beta_{2}}\right), \\
& Q_{2}=\alpha \sigma_{2}\left(e^{\beta_{2}}-e^{\beta_{1}}\right) \\
& Z_{1}=-\frac{P_{1}+P_{2}}{i N^{2}+M^{2} R+\frac{1}{D a}}+\frac{\alpha}{\sigma_{1}} R_{1} \\
& Z_{2}=-\frac{P_{3}+P_{4}}{i N^{2}+M^{2} R+\frac{1}{D a}}-\frac{\alpha}{\sigma_{2}} R_{1}
\end{aligned}
$$

Collecting the real and imaginary part, this in virtue of (41) reduces to

$$
\tilde{u}=\left(\varphi_{1} \cos \omega t-\varphi_{2} \sin \omega t\right)+i\left(\varphi_{2} \cos \omega t+\varphi_{1} \sin \omega t\right)
$$

where the coefficients $\varphi_{1}$ and $\varphi_{2}$ are obtained but not listed here for simplicity. The above equations describe the steady and unsteady fluid velocity profile in the general case. Many particular results appear emerge easily from the above solutions which have been discussed in next section.

\section{DEDUCTIONS}

5.1 If the permeability of upper and lower beds is equal ( $\left.\tilde{i}_{*} \epsilon_{x} \sigma_{1}=\sigma_{2}=\sigma\right)$, both the steady and unsteady velocity profiles reduce from the general case to:

Steady Part

$\tilde{u}=C_{1} e^{\left(\alpha_{1} y\right)}+C_{2} e^{\left(\alpha_{2} y\right)}+\frac{R}{M^{2} R+1 / D a}$

where $\alpha_{1,2}=\frac{1}{2}\left[R \pm \sqrt{ }\left(R^{2}+4\left(M^{2} R+\frac{1}{D a}\right)\right)\right]$,

$$
C_{1}=\frac{1}{e^{\alpha_{2}}-e^{\alpha_{1}}}\left[-\bar{u}_{B 2}+e^{\alpha_{2}} \bar{u}_{B 1}-\frac{R}{\left(M^{2} R+\frac{1}{D a}\right)}\left(e^{\alpha_{2}}-1\right)\right]
$$

$$
C_{2}=\frac{1}{e^{\alpha_{2}}-e^{\alpha_{1}}}\left[\bar{u}_{B 2}-e^{\alpha_{1}} \bar{u}_{B 1}-\frac{R}{\left(M^{2} R+\frac{1}{D a}\right)}\left(1-e^{\alpha_{1}}\right)\right]
$$

And the slip velocities are given by

$\bar{u}_{B 1}=\frac{D_{2} E_{2}-D_{4} E_{1}}{D_{1} D_{4}-D_{3} D_{2}}$

$\bar{u}_{B 2}=\frac{D_{3} E_{1}-D_{1} E_{2}}{D_{1} D_{4}-D_{3} D_{2}}$

Where

$D_{1}=\frac{\alpha_{1} e^{\alpha_{2}}-\alpha_{2} e^{\alpha_{1}}}{e^{\alpha_{2}}-e^{\alpha_{1}}}-\alpha \sigma, D_{2}=\frac{\alpha_{2}-\alpha_{1}}{e^{\alpha_{2}}-e^{\alpha_{1}}}$

$D_{3}=\frac{\left(\alpha_{1}-\alpha_{2}\right) e^{\alpha_{1}+\alpha_{2}}}{e^{\alpha_{2}}-e^{\alpha_{1}}}, D_{4}=\frac{\alpha_{2} e^{\alpha_{2}}-\alpha_{1} e^{\alpha_{1}}}{e^{\alpha_{2}}-e^{\alpha_{1}}}+\alpha \sigma$,

$E_{1}=R\left[\frac{1}{\left(M^{2} R+\frac{1}{D a}\right)}\left\{\frac{\alpha_{2}\left(e^{\alpha_{1}}-1\right)}{e^{\alpha_{2}}-e^{\alpha_{1}}}-\frac{\alpha_{1}\left(e^{\alpha_{2}}-1\right)}{e^{\alpha_{2}}-e^{\alpha_{1}}}\right\}+\frac{\alpha}{\sigma}\right]$

$E_{2}=R\left[\frac{1}{\left(M^{2} R+\frac{1}{D a}\right)}\left\{\frac{\alpha_{2} e^{\alpha_{2}}\left(e^{\alpha_{1}}-1\right)}{e^{\alpha_{2}}-e^{\alpha_{1}}}-\frac{\alpha_{1} e^{\alpha_{1}}\left(e^{\alpha_{2}}-1\right)}{e^{\alpha_{2}}-e^{\alpha_{1}}}\right\}-\frac{\alpha}{\sigma}\right.$

Unsteady Part

$\tilde{u}=\tilde{f}(y) e^{i \omega t}=\left\{A_{1} e^{\beta_{1} y}+A_{2} e^{\beta_{2} y}+\frac{1}{i N^{2}+M^{2} R+\frac{1}{D a}}\right\} e^{i \omega t}$

where $\beta_{1,2}=\frac{1}{2}\left[R \pm \sqrt{ }\left(R^{2}+4\left(i N^{2}+M^{2} R+\frac{1}{D a}\right)\right)\right]$,

$N^{2}=\omega$.

$A_{1}=\frac{1}{e^{\beta_{2}}-e^{\beta_{1}}}\left[-\frac{e^{\beta_{2}}-1}{i N^{2}+M^{2} R+\frac{1}{D a}}-\tilde{f_{2}}+\tilde{f_{1}} e^{\beta_{2}}\right]$

$A_{2}=\frac{1}{e^{\beta_{2}}-e^{\beta_{1}}}\left[\frac{e^{\beta_{1}}-1}{i N^{2}+M^{2} R+\frac{1}{D a}}+\tilde{f_{2}}-\tilde{f_{1}} e^{\beta_{1}}\right]$

And the slip velocities are given by 


$$
\begin{aligned}
& \tilde{u}_{B 1}=\tilde{f_{1}} e^{i \omega t}=\frac{P_{2} Z_{2}-Z_{1}\left(P_{4}+Q_{2}\right)}{\left(P_{1}+Q_{1}\right)\left(P_{4}+Q_{2}\right)-P_{3} P_{2}} e^{i \omega t} \\
& \tilde{u}_{B 2}=\tilde{f_{2}} e^{i \omega t}=\frac{P_{3} Z_{1}-Z_{2}\left(P_{1}+Q_{1}\right)}{\left(P_{1}+Q_{1}\right)\left(P_{4}+Q_{2}\right)-P_{3} P_{2}} e^{i \omega t}
\end{aligned}
$$

where

$$
\begin{aligned}
& P_{1}=\left(\beta_{1} e^{\beta_{2}}-\beta_{2} e^{\beta_{1}}\right), P_{2}=\beta_{2}-\beta_{1}, \\
& P_{3}=e^{\beta_{1}+\beta_{2}}\left(\beta_{1}-\beta_{2}\right), P_{4}=\left(\beta_{2} e^{\beta_{2}}-\beta_{1} e^{\beta_{1}}\right), \\
& R_{1}=\left(e^{\beta_{2}}-e^{\beta_{1}}\right), Q_{1}=\alpha \sigma\left(e^{\beta_{1}}-e^{\beta_{2}}\right), \\
& Q_{2}=\alpha \sigma\left(e^{\beta_{2}}-e^{\beta_{1}}\right), \\
& Z_{1}=-\frac{P_{1}+P_{2}}{i N^{2}+M^{2} R+\frac{1}{D a}}+\frac{\alpha}{\sigma} R_{1}, \\
& Z_{2}=-\frac{P_{3}+P_{4}}{i N^{2}+M^{2} R+\frac{1}{D a}}-\frac{\alpha}{\sigma} R_{1} .
\end{aligned}
$$

\subsection{Mass Flow}

The instantaneous Mass Flux $Q$ of unsteady velocity field is given by

$$
\begin{aligned}
& Q=\left[\int_{0}^{1} \tilde{f}(y) d y\right] e^{i \omega t}=\frac{1}{\beta_{1} \beta_{2}\left(e^{\beta_{2}}-e^{\beta_{1}}\right)} \\
& {\left[\beta_{2} e^{i \omega t}\left\{\left(e^{\beta_{1}+\beta_{2}}-e^{\beta_{2}}\right) \tilde{f}_{1}+\left(1-e^{\beta_{1}}\right) \tilde{f}_{2}+\frac{1}{i N^{2}+M^{2} R+\frac{1}{D a}}\left(1-e^{\beta_{1}}\right)\left(e^{\beta_{2}}-1\right)\right\}\right.} \\
& \left.\beta_{1} e^{i \omega t}\left\{\left(e^{\beta_{1}}-e^{\beta_{1}+\beta_{2}}\right) \tilde{f}+\left(e^{\beta_{2}}-1\right) \tilde{f} 2+\frac{1}{i N^{2}+M^{2} R+\frac{1}{D a}}\left(e^{\beta_{1}}-1\right)\left(e^{\beta_{2}}-1\right)\right\}\right] \\
& +\frac{e^{i \omega t}}{i N^{2}+M^{2} R+\frac{1}{D a}}
\end{aligned}
$$

\subsection{Shear Stress}

Although One can easily obtain the shear stress $\tilde{\tau}$ at the walls using

$$
\tilde{\tau}=\left(\frac{\tau}{\rho h B_{1}}\right)=\frac{\partial \tilde{u}}{\partial y}
$$

\section{RESULTS AND DISCUSSION}

Numerical evaluations have been performed and some graphical results have been obtained to illustrate the details of the flow characteristics. Figures 2 and 3 reveals the effect of an increase in permeability parameter $\sigma$ and Hartmann number $M$ on the steady state velocity profile. It is seen that an increase in permeability parameter $\sigma$ and Hartmann number $M$ leads to a decrease in the steady state velocity. It can be also observed that for fixed $\sigma$ and $M$ the velocity increases with increase in $y$ initially and attains a maximum value slightly away from the middle of permeable beds and is in the neighborhood of point $y=0.6$. After achieving maximum value, the velocity decreases with the increment iny. Fig. 4 depicts the influence of Darcy number $D a$ on the steady state velocity profile. As $D a$ increases from 0.01 to 100 , a considerable increment in steady state velocity occurs.

The unsteady velocity distributions for various values of the significant parameters are illustrated in Figs. 510. It can be seen from Fig. 5 that the velocity is greater when $\omega t$ is minimum. It is interesting to note that the velocity can attain its maximum even at the lower permeable bed for some specific choice of parameters $\left(\omega t=3 \pi / 4, \omega t=\pi\right.$ for $\left.a={ }_{\star} 1\right)$. Figure 6 explains that velocity decreases as $\sigma$ increases and the maximum velocity occurs towards the upper bed. Figures 7 and 8 exhibit the effect of an increase in Hartmann number $M$ on the velocity profile. As $M$ increases from 0 to 3 , a considerable reduction in the unsteady state velocity occurs. It is because that the application of transverse magnetic field will result a resistive type force (Lorentz force) similar to drag force which tends to resist the fluid and thus reducing its velocity. Moreover, for fixed $M$, the velocity increases as $y$ increases initially and attains the maximum velocity towards the upper bed then velocity decreases with the increment in $y$. But in the case of $M=1.5,2$ and 3 with $R=10$, velocity decreases initially with the increment in $y$. 
R. Kumar and B. G. Prasad / JAFM, Vol. 7, No. 1, pp. 63-74, 2014.

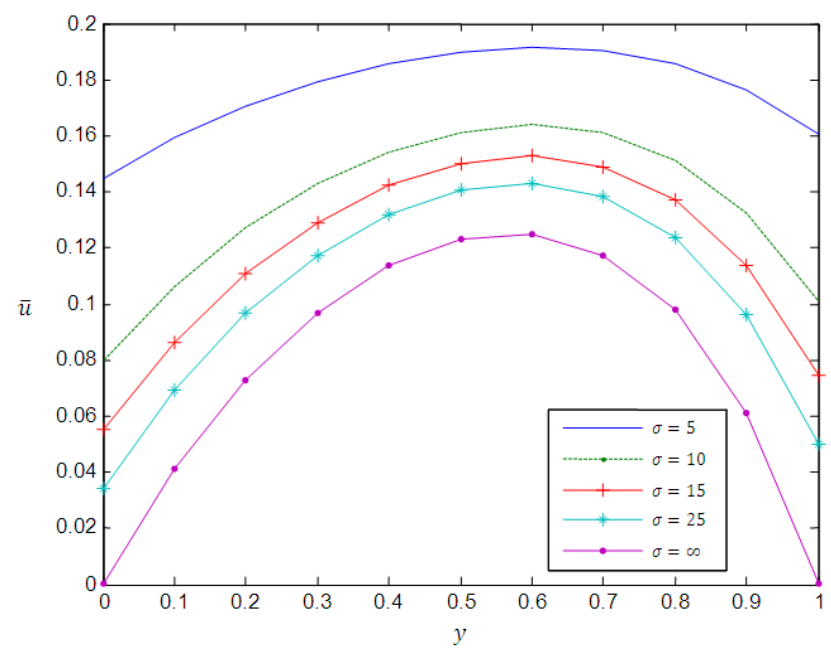

Fig. 2. Steady state velocity profiles for $a=5_{w} R=2, M=2$ and $D a=1$

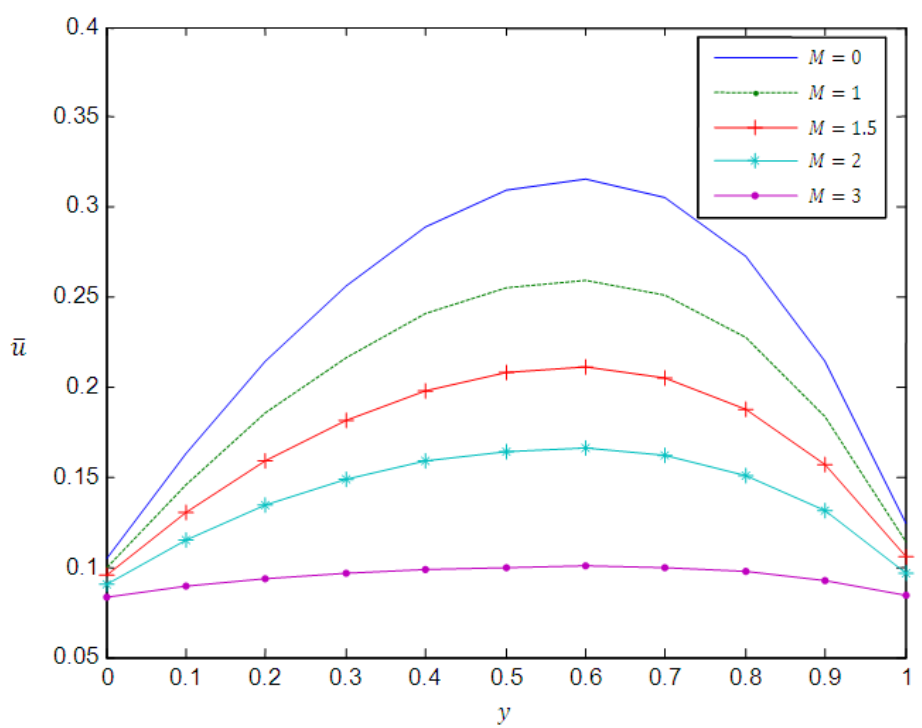

Fig. 3. Steady state velocity profiles for $\alpha=5_{w} R=2_{y} \sigma=5$ and $D a=1$

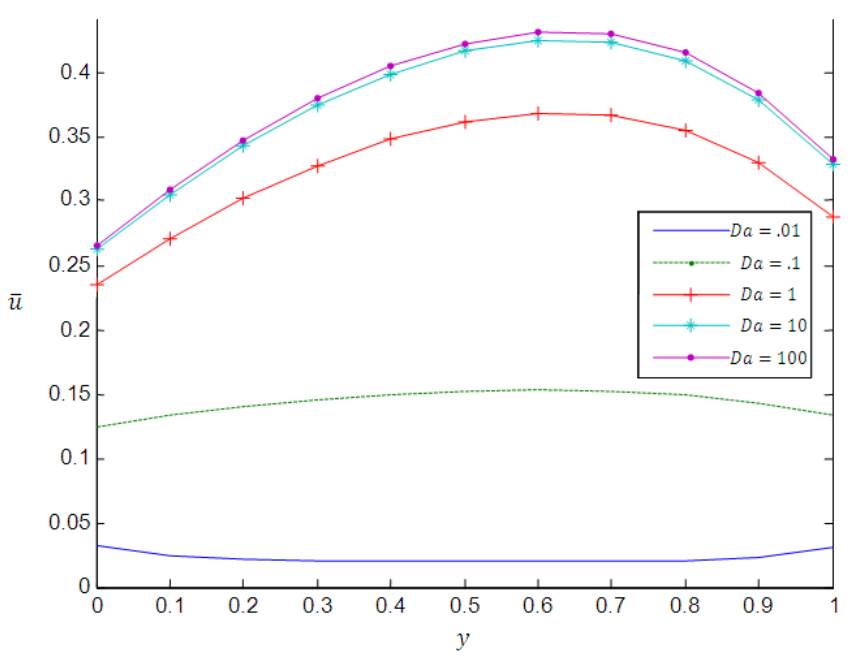

Fig. 4. Steady state velocity profiles for $\alpha={ }_{w} R=2{ }_{v} \sigma=5$ and $M=1$ 
As a fact the presence of a porous medium increases the resistance to flow resulting in decrease in the fluid velocity. Fig. 9 shows that the velocity increases gradually with increase of $D a$ value which is as expected. The maximum velocity is shifted towards the upper bed whereas the point of maximum is found slightly distant from the middle of the beds

Figure 10 reveals the effect of an increase in wt on the mass flow. We observe that the mass flow decreases gradually with increase of wt value which is as expected. Figure 11 shows that with the increase of Darcy number $D a$ the mass flow decreases. It can be also seen that for a particular value of $D a$, the mass flow decreases with the increment in Reynolds number $R$.

Tables 1(A) and (B) represent the values of the shear stress at the lower and upper walls for $\alpha=0.5{ }_{x} \sigma=5_{x} R=5_{x} M=0, N=1$ and for different values of $\mathrm{Da}$. We observe that an increase in Darcy number $D a$, leads to a decrease in the stress on the lower wall and increase on the upper wall. Also it is seen that for a particular value of $D a$, the stress at the lower wall of the permeable bed is lower than that of the upper permeable bed. The effect of the cross-flow Reynolds number on the values of shear stress on the permeable walls are exhibited in Table $1(\mathrm{C})$ for different values of the cross-flow Reynolds number $R$. It is obvious that as the cross-flow Reynolds number increases the shear stress on both the lower and upper walls decreases. Further, when $R=0$, stress takes the maximum value at lower wall but for upper wall it attains the maximum value at $R=2$. Table 1(D) presents the values of the shear stress for $\alpha=0.5, \sigma=10, R=5_{z} D a^{-1}=.1$ and for different values of Hartmann number $M$. We find that the shear stress at both the lower and upper walls of the permeable bed increases with the increase of $M$. Table

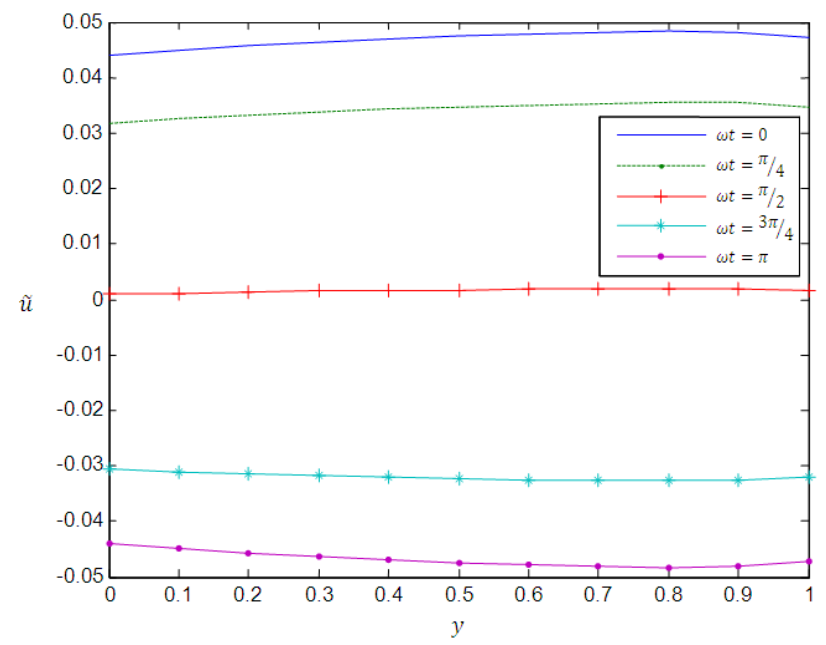

Fig. 5. Unsteady state velocity profiles for $\alpha={ }^{5} 5_{v} R=10_{v} \sigma=5_{v} N=1_{v} D a={ }_{x} 1$ and $M=1$

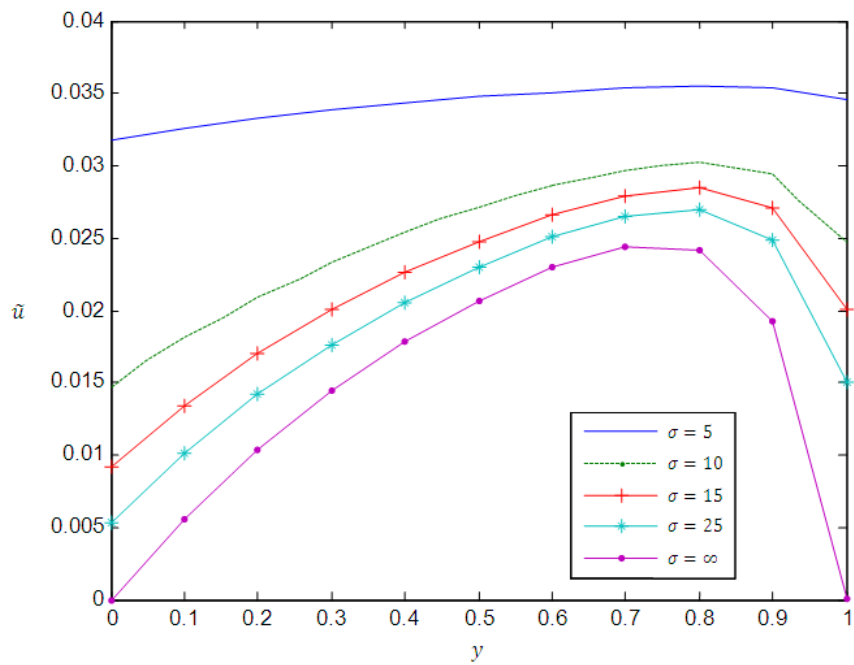

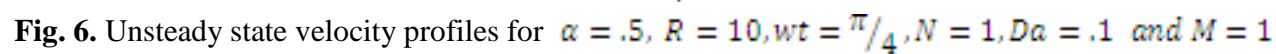


R. Kumar and B. G. Prasad / JAFM, Vol. 7, No. 1, pp. 63-74, 2014.

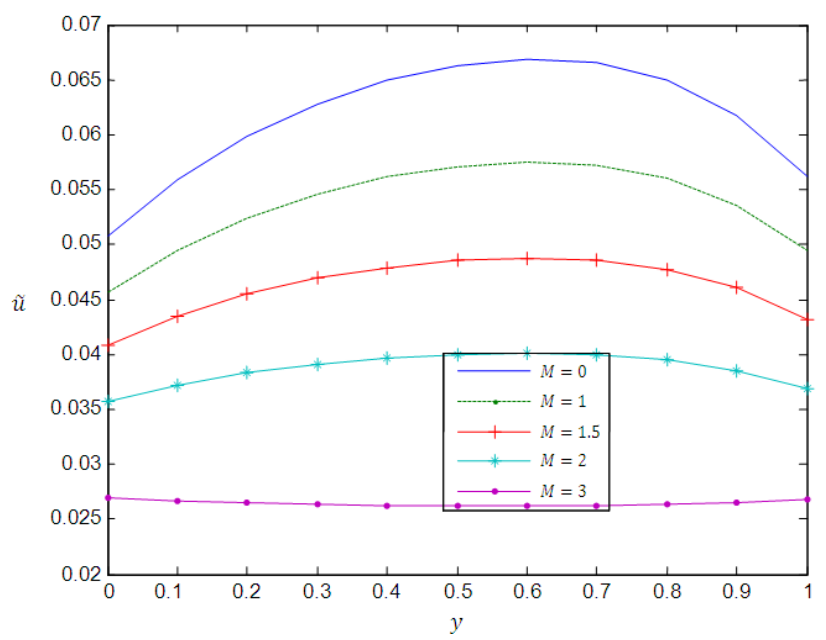

Fig. 7. Unsteady state velocity profiles for $\mathbb{a}={ }_{a}, R=2_{v} W t={ }^{\pi} f_{4} N=1_{v} \sigma=5$ and $D a={ }_{\alpha} 1$

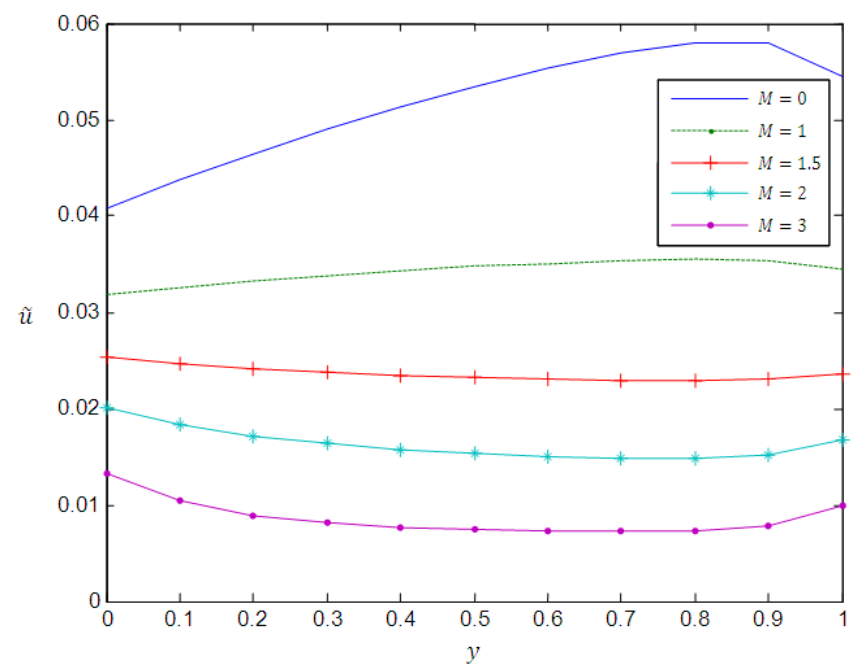

Fig. 8. Unsteady state velocity profiles for $\alpha={ }_{n} R=10_{v} \omega t={ }^{\pi}{ }_{4}{ } N=1_{v} \sigma=5$ and $D a={ }_{x} 1$

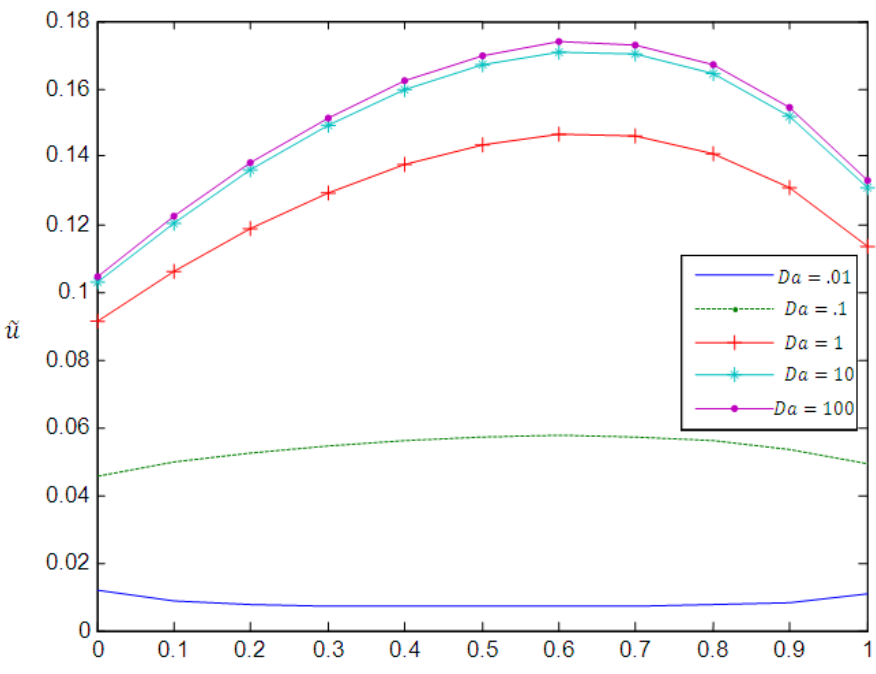

Fig. 9. Unsteady state velocity profiles for $\alpha=5_{v} R=2_{v} W t=\pi_{4} W=1_{0} \sigma=5$ and $M=1$ 
R. Kumar and B. G. Prasad / JAFM, Vol. 7, No. 1, pp. 63-74, 2014.

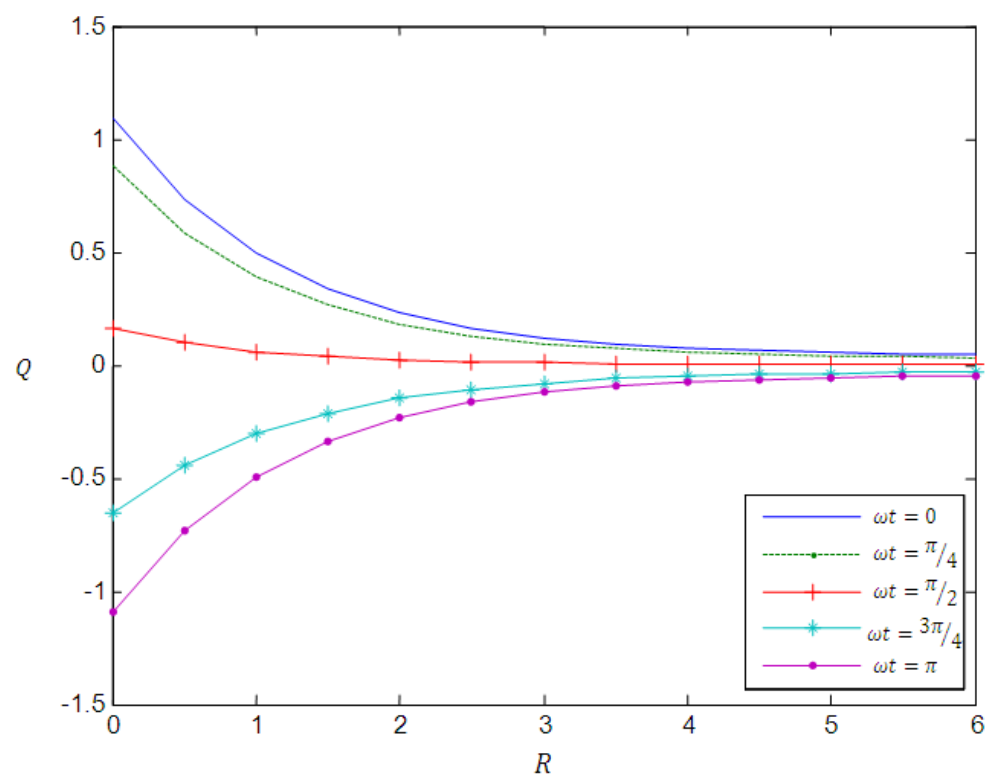

Fig. 10. Mass flux flow for $\mathbb{Q}={ }_{a} \mathrm{Da}={ }_{\mathrm{r}}{ }_{\mathrm{W}} N=1_{\mathrm{v}} \sigma=5$ and $M=1$

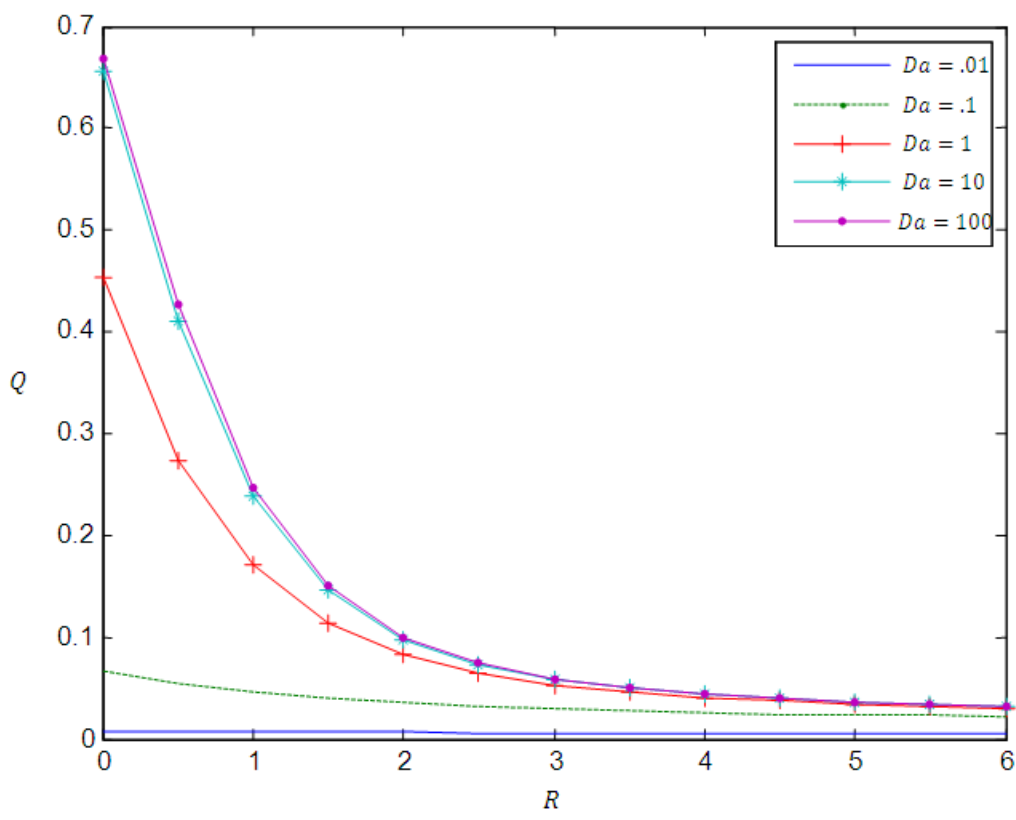

Fig. 11. Mass flux flow for $\alpha=5_{w} \omega t=\pi_{4} N=1_{w} \sigma=25$ and $M=1$

1(E) exhibits the values of the shear stress for $\alpha=0.5, \sigma=10, R=5, D a={ }_{\alpha} 1, M=2$ and for different values of frequency parameter $N$. We find that the stress on the lower wall of the permeable bed increases with the increase of $M$ while on the upper wall it decreases with the increment of $M$. The effect of an increase in the permeability parameter, $\sigma_{2}$ on the shear stress on the walls of permeable beds is presented in Table 1(F). It can be observed that the shear stress on both the lower and upper walls of the permeable beds increase with an increase of permeability parameter. Further, when these observations are compared with the findings in Malathi and Srinivas (2008) for the clear fluid case and Vajravelu et al. (2003) for the clear fluid case and the absence of magnetic field we find very good agreement. 
R. Kumar and B. G. Prasad / JAFM, Vol. 7, No. 1, pp. 63-74, 2014.

Table 1 Variation of shear stress

\begin{tabular}{|c|c|c|c|c|c|}
\hline \multirow{3}{*}{$\begin{array}{l}\text { (A): } \alpha= \\
\left\|\frac{\tau}{\tau}\right\|\end{array}$} & \multirow{2}{*}{\multicolumn{5}{|c|}{$\begin{array}{l}0.5, \sigma \\
D Q \\
\end{array}$}} \\
\hline & & & & & \\
\hline & 0.01 & 0.1 & 1 & 10 & 100 \\
\hline $\begin{array}{l}\text { Lower } \\
\text { wall }\end{array}$ & 0.0623 & 0.0297 & 0.0217 & 0.0208 & 0.0207 \\
\hline $\begin{array}{l}\text { Upper } \\
\text { wall }\end{array}$ & 0.0720 & 0.0417 & 0.0320 & 0.0309 & 0.0307 \\
\hline \multicolumn{6}{|c|}{ (B): $\alpha=0.5, \sigma=5, M=0, R=10, \mathbb{N}=1$} \\
\hline \multirow[b]{2}{*}{$\|\tilde{T}\|$} & \multicolumn{5}{|c|}{$D_{Q}$} \\
\hline & 0.01 & 0.1 & 1 & 10 & 100 \\
\hline $\begin{array}{l}\text { Lower } \\
\text { wall }\end{array}$ & 0.0534 & 0.0403 & 0.0914 & 0.0990 & 0.0998 \\
\hline $\begin{array}{l}\text { Upper } \\
\text { wall }\end{array}$ & 0.0649 & 0.0834 & 0.2311 & 0.2544 & 0.2594 \\
\hline \multicolumn{6}{|c|}{ (C): $\alpha=0.5, \sigma=5, M=0, D a=1, N=1$} \\
\hline \multirow[b]{2}{*}{$\|\tilde{\tau}\|$} & \multicolumn{5}{|c|}{$\mathrm{R}$} \\
\hline & 0 & 2 & 4 & 6 & 8 \\
\hline $\begin{array}{l}\text { Lower } \\
\text { wall }\end{array}$ & 0.3657 & 0.2805 & 0.1983 & 0.1450 & 0.1124 \\
\hline $\begin{array}{l}\text { Upper } \\
\text { wall }\end{array}$ & 0.3657 & 0.3896 & 0.3548 & 0.3067 & 0.2647 \\
\hline \multicolumn{6}{|c|}{ (D): $\alpha=0.5, \sigma=5, R=10, D Q=0.1, N=1$} \\
\hline \multirow[b]{2}{*}{$\|\tilde{\tau}\|$} & \multicolumn{5}{|c|}{$\mathrm{M}$} \\
\hline & 2 & 4 & 6 & 8 & 10 \\
\hline $\begin{array}{l}\text { Lower } \\
\text { wall }\end{array}$ & 0.0297 & 0.0667 & 0.0798 & 0.0859 & 0.0893 \\
\hline $\begin{array}{l}\text { Upper } \\
\text { wall } \\
\end{array}$ & 0.0417 & 0.0754 & 0.0847 & 0.0890 & 0.0914 \\
\hline \multicolumn{6}{|c|}{ (E): $\alpha=0.5, \sigma=5, \mathbb{R}=5, D_{\alpha}=0.1, \quad M=2$} \\
\hline \multirow[b]{2}{*}{$\|\tilde{\pi}\|$} & \multicolumn{5}{|c|}{$N$} \\
\hline & 1 & 2 & 3 & 4 & 5 \\
\hline $\begin{array}{l}\text { Lower } \\
\text { wall }\end{array}$ & 0.0099 & 0.0124 & 0.0196 & 0.0304 & 0.0424 \\
\hline $\begin{array}{l}\text { Upper } \\
\text { wall }\end{array}$ & 0.0806 & 0.0801 & 0.0785 & 0.0770 & 0.0767 \\
\hline \multicolumn{6}{|c|}{$(\mathrm{F}): \alpha=0.5, \mathbb{N}=1, R=5, D_{Q}=1_{x} M=1$} \\
\hline \multirow[b]{2}{*}{$\|\tilde{\tau}\|$} & \multicolumn{5}{|l|}{$\sigma$} \\
\hline & 2 & 4 & 6 & 8 & 10 \\
\hline $\begin{array}{l}\text { Lower } \\
\text { wall }\end{array}$ & 0.0463 & 0.0693 & 0.1035 & 0.1200 & 0.1296 \\
\hline $\begin{array}{l}\text { Upper } \\
\text { wall }\end{array}$ & 0.0624 & 0.1117 & 0.1879 & 0.2374 & 0.2738 \\
\hline
\end{tabular}




\section{REFERENCES}

Beavers, G.S. and D.D. Joseph (1967). Boundary conditions at a naturally permeable wall, J. Fluid Mech., 30 197-206.

Berman, A.S. (1958). Laminar flow in an annulus with porous walls, J. Appl. Phys. ,29, 71-75.

Chamkha, A. J. (1997). MHD-free convection from a vertical plate embedded in a thermally stratified porous medium with Hall effects, Appl. Math. Modeling, 21, 603-609.

Chamkha, A. J. (1999). Magnetohydrodynamic mixed convection from a rotating cone embedded in a porous medium with heat generation, J. Porous Media, 2, 87-105.

Chamkha, A. J. and A. Abdul-Rahim Khaled (2000). Hydromagnetic combined heat and mass transfer by natural convection from a permeable surface embedded in a fluid saturated porous medium, Int . J. Num. Meth. Heat Fluid Flow, 10(5), 455-476.

Chandra, P. and J.S.V.R.K. Prasad (1994). Pulsatile flow in circular tubes of varying cross-section with suction/injection, J. Aust. Math. Soc. Ser. B, 35, 366-381.

Hasan, A.B.M. Toufique and D. K. Das (2008) Numerical Simulation of Sinusoidal Fluctuated Pulsatile Laminar Flow through Stenotic Artery, $J$. Appl. Fluid Mech., 1(2), 25-35.

Khodadadi, J .M. (1991). Oscillatory fluid flow through a porous medium channel bounded by two impermeable parallel plates, J. Fluid Eng., 113, 509-511.

Kim, S.Y., B. H. Kang and J. M. Hyun (1994). Heat transfer from pulsating flow in a channel filled with porous media, Int. J. Heat Mass Trans., 37, 2025-2033.

Malathy, T., S. Srinivas (2008). Pulsatile flow of a hydromagnetic fluid between permeable beds, Int Commun. Heat Mass Trans., 35, 681-688.

Radhakrishnamacharya, G. and M.K. Maiti (1977). Heat transfer to pulsatile flow in a porous channel, Int. J. Heat Mass Trans., 20, 171-173.

Raptis, A. and C. Perdikis (1983). MHD unsteady free convection flow through a porous medium, Energy Res., 7, 391-395.

Raptis, A., C. Massias and G. Tzivanidis (1982). Hydromagnetic free convection flow through a porous medium between two parallel plates, Phys. Lett., 90A, 288-289.

Uchida, S. (1956). The pulsating viscous flow superposed on the steady laminar motion of incompressible fluid in a circular pipe, J. Appl. Math. Phys. (ZAMP), 7, 403-422.

Vajravelu, K., K. Ramesh, S. Sreenadh, P.V. Arunachalam (2003). Pulsatile flow between permeable beds, Int. J. Non-Linear Mech., 38, 999-1005.

Wang, Y.C. (1971). Pulsatile flow in a porous channel, Trans ASME, J. Appl. Mech., 38, 553-555. 